\title{
Quasi-Cut of Fuzzy Sets and Quasi-Cut of Instuitionistic Fuzzy Sets
}

\author{
S. R. Barbhuiya \\ Srikishan Sarda College, Hailakandi \\ Hailakandi-788151, Assam, India
}

\begin{abstract}
The aim of this paper is to study the properties of t-cut set, strong t-cut set, t-quasi-cut set, strong t-quasi-cut set and $\in V q$-cut set of a fuzzy set $\mu$. For any intuitionistic fuzzy $A=\left\langle\mu_{A}, \nu_{A}>\right.$ and $\alpha, \beta \in[0,1]$, we define and study the properties of upper $(\alpha, \beta)$ cut set $A_{(\alpha, \beta)}$, strong upper $(\alpha, \beta)$ cut set $A_{(\alpha, \beta)}$, lower $(\alpha, \beta)$ cut set $A^{(\alpha, \beta)}$, strong lower $(\alpha, \beta)$ cut set $A^{(\alpha, \beta)}$, upper $(\alpha, \beta)$-quasi-cut set $A_{<\alpha, \beta>}$, strong upper $(\alpha, \beta)$-quasi-cut set $A_{<\alpha, \beta>}$, lower $(\alpha, \beta)$-quasi-cut set $A^{<\alpha, \beta>}$, strong lower $(\alpha, \beta)$-quasi-cut set $A^{<\alpha, \beta>}$ and $\in \vee q$-cut set.
\end{abstract}

\section{Keywords}

Cut set, Strong cut set, Quasi cut set, Strong quasi cut set, $(\alpha, \beta)$ cut set, Upper $(\alpha, \beta)$-cut set, Strong upper $(\alpha, \beta)$-cut set, Lower $(\alpha, \beta)$-cut set, Strong lower $(\alpha, \beta)$-cut set, $\operatorname{Upper}(\alpha, \beta)$-quasicut set, Lower $(\alpha, \beta)$-quasi-cut set, Strong upper $(\alpha, \beta)$-quasi-cut set, Strong lower $(\alpha, \beta)$-quasi-cut set, $\in \vee q$-cut set.

AMS Subject Classification(2010):03E72, 03F55;

\section{INTRODUCTION}

In many complicated situations in real life several types of uncertainties occur, to handle such situation we have theory of Probability, theory of Interval Mathematics, Fuzzy set theory, Rough set theory and Vague set theory. Fuzzy set is introduced in [1] by Zadeh. The theory of fuzzy set is further generalised to intuitionistic fuzzy set, interval valued fuzzy set, temporal intuitionistic fuzzy set etc. The concept of intuitionistic fuzzy set introduced by Atanassov [2] in 1983. Das [3] introduced the notion of level subset (called cut set ) of a set. Since then different researcher [4, 5, 6, 3, 7, have contributed significantly for the development of literatures of cut sets. In the theory of fuzzy sets, intuitionistic fuzzy sets, interval valued fuzzy sets, cut sets play very important rule for the development of the theory. Here in this paper, various types of cut sets in fuzzy sets and in intuitionistic fuzzy sets are discussed.

\section{PRELIMINARIES}

DEFINITION 1. (4] 5]) A fuzzy set $\mu$ of the form $\mu(y)= \begin{cases}t, & \text { if } y=x \quad t \in(0,1] \\ 0, & \text { if } y \neq x\end{cases}$

is called a fuzzy point with support $x$ and value $t$ and is denoted by $x_{t}$.

DEFINITION 2. (4] 57) A fuzzy point $x_{t}$ is said to belong to (respectively be quasi coincident with) a fuzzy set $\mu$ written as $x_{t} \in$ $\mu$ (respectively $\left.x_{t} q \mu\right)$ if $\mu(x) \geq t$ (respectively $\left.\mu(x)+t>1\right)$. If $x_{t} \in \mu$ or $x_{t} q \mu$, then we write $x_{t} \in \vee q \mu$. (Note $\overline{\in \vee}$ means $\in \vee q$ does not hold).

Definition 3. Let $X$ be a set and $\mu$ be a fuzzy subset of $X$, then $t$-cut set and $t$-strong cut set of fuzzy set $\mu$ are given by

$\mu_{t}=\{x \mid x \in X$ and $\mu(x) \geq t\}$ and

$\mu_{t}=\{x \mid x \in X \quad$ and $\mu(x)>t\}$

From the point of view of neighbourhood, we have $x \in \mu_{t} \Leftrightarrow$ $\mu(x) \geq t \Leftrightarrow x_{t} \in \mu$. Prof.L. Cheng-Zhong [8]introduced a new concept of strong neighbourhood and he define $x \in \mu_{t} \Leftrightarrow \mu(x)>$ $t \Leftrightarrow x_{t} \in \mu$

Therefore $t$-cut set and $t$-strong cut set of fuzzy set $\mu$ are given by $\mu_{t}=\left\{x \mid x_{t} \in \mu\right\} \quad \mu_{t}=\left\{x \mid x_{t} \in \mu\right\}$

Quasi neighbourhood play an important rule in fuzzy topology [11. 12]. Now $x_{t} q \mu \Leftrightarrow \mu(x)+t>1$ based on quasi neighbourhood, we can defined a new kind of cut set as $<\mu>_{t}=$ $\left\{x \quad \mid x_{t} q \mu\right\}$ Here $\left\langle\mu>_{t}\right.$ is called as $t$-strong quasi-cut set of fuzzy set $\mu$. Combining both the notion we define another cut set as $[\mu]_{t}=\left\{x \mid x_{t} \in \vee q \mu\right\}$. Here $[\mu]_{t}$ is called $\in \vee q$-cut set.

Definition 4. Let $X$ be a set and $\mu$ be a fuzzy subset of $X$, then $t$-quasi-cut set and $t$-strong quasi cut set of fuzzy set $\mu$ are are denoted and defined by

$$
<\mu>_{t}=\{x \mid x \in X \quad \mu(x)+t \geq 1\}=\left\{x \mid x_{t} q \mu\right\}
$$$$
<\mu>_{t}=\{x \mid x \in X \quad \mu(x)+t>1\}=\left\{x \mid x_{t} q \mu\right\}
$$

Definition 5. ([9] [10]) An intuitionistic fuzzy set (IFS) $A$ of a BG-algebra $X$ is an object of the form $A=\{<$ $\left.x, \mu_{A}(x), \nu_{A}(x)>\mid x \in X\right\}$, where $\mu_{A}: X \rightarrow[0,1]$ and $\nu_{A}: X \rightarrow[0,1]$ with the condition $0 \leq \mu_{A}(x)+\nu_{A}(x) \leq$ $1, \forall x \in X$. The numbers $\mu_{A}(x)$ and $\nu_{A}(x)$ denote respectively the degree of membership and the degree of non-membership of the element $x$ in set $A$. For the sake of simplicity, we shall use the symbol $A=\left(\mu_{A}, \nu_{A}\right)$ for the intuitionistic fuzzy set $A=\{<$ 
$\left.x, \mu_{A}(x), \nu_{A}(x)>\mid x \in X\right\}$. The class of IFSs on a universe $X$ is denoted by IF $S(X)$.

Definition 6. ([4]) If $A=\left\{<x, \mu_{A}(x), \nu_{A}(x)>\mid x \in X\right\}$ and $\left.B=\left\{<x, \mu_{B}(x), \nu_{B}(x)\right\rangle \mid x \in X\right\}$ are any two IFS of $a$ set $X$, then

$A \subseteq B$ if and only if for all $x \in X, \mu_{A}(x) \leq \mu_{B}(x)$ and $\nu_{A}(x) \geq \nu_{B}(x)$

$A=B$ if and only if for all $x \in X, \mu_{A}(x)=\mu_{B}(x)$ and $\nu_{A}(x)=\nu_{B}(x)$,

$A \cap B=\left\{\left\langle x,\left(\mu_{A} \cap \mu_{B}\right)(x),\left(\nu_{A} \cup \nu_{B}\right)(x)>\right| x \in X\right\}$,

where $\left(\mu_{A} \cap \mu_{B}\right)(x)=\min \left\{\mu_{A}(x), \mu_{B}(x)\right\}$ and $\left(\nu_{A} \cup \nu_{B}\right)(x)=\max \left\{\nu_{A}(x), \nu_{B}(x)\right\}$,

$A \cup B=\left\{\left\langle x,\left(\mu_{A} \cup \mu_{B}\right)(x),\left(\nu_{A} \cap \nu_{B}\right)(x)\right\rangle \mid x \in X\right\}$,

where $\left(\mu_{A} \cup \mu_{B}\right)(x)=\max \left\{\mu_{A}(x), \mu_{B}(x)\right\}$ and $\left(\nu_{A} \cap \nu_{B}\right)(x)=\min \left\{\nu_{A}(x), \nu_{B}(x)\right\}$

DEFINITION 7. Let $X$ and $Y$ be two non empty sets and $f: X \longrightarrow Y$ be a mapping. Let $\mu$ and $\nu$ be two fuzzy subsets of $X$ and $Y$ respectively. Then the image of $\mu$ under the map $f$ is denoted by $f(\mu)$ and is defined by $f(\mu)(y)=\left\{\begin{array}{l}\vee\left\{\mu(x): x \in f^{-1}(y) \neq \Phi\right\} \\ 0 \quad \text { otherwise }\end{array}\right.$

also pre image of $\nu$ under $f$ is denoted by $f^{-1}(\nu)$ and is defined as $\left.f^{-1}(\nu)(x)=\nu(f(x))\right) ; \forall x \in X$

DEFINITION 8. Let $X$ and $Y$ be two non empty sets and $f: X \longrightarrow Y$ be a mapping. Let $A$ and $B$ be IFS's of $X$ and $Y$ respectively. Then the image of $A$ under the map $f$ is denoted by $f(A)$ and is defined by $f(A)(y)=\left(\mu_{f(A)}(y), \nu_{f(A)}(y)\right)$, where

$\mu_{f(A)}(y)=\left\{\begin{array}{l}\vee\left\{\mu_{A}(x): x \in f^{-1}(y) \neq \Phi\right\} \\ 0 \quad \text { otherwise }\end{array}\right.$ and

$\nu_{f(A)}(y)=\left\{\begin{array}{l}\wedge\left\{\nu_{A}(x): x \in f^{-1}(y) \neq \Phi\right\} \\ 1 \quad \text { otherwise }\end{array}\right.$

also pre image of $B$ under $f$ is denoted by $f^{-1}(B)$ and is defined as $f^{-1}(B)(x)=\left(\mu_{f^{-1}(B)}, \nu_{f^{-1}(B)}\right)=$ $\left(\mu_{B}(f(x)), \nu_{B}(f(x))\right) ; \forall x \in X$

$\mu_{A}(x) \leq \mu_{f(A)}(f(x))$ and $\nu_{A}(x) \geq \nu_{f(A)}(f(x)) \quad \forall x \in X$ however equality hold when the map $\mathrm{f}$ is bijective.

DEFINITION 9. [4. 57 A fuzzy point $x_{t}$ is said to belong to (respectively be quasi coincident with) an intuitionistic fuzzy set $A=\left\{\left\langle x, \mu_{A}(x), \nu_{A}(x)\right\rangle \mid x \in X\right\}$ written as $x_{t} \in A$ (respectively $\left.x_{t} q A\right)$, if $\mu_{A}(x) \geq t$ (respectively $\mu_{A}(x)+t>1$ ) and $\nu_{A}(x) \leq t$ (respectively $\left.\nu_{A}(x)+t<1\right)$. If $x_{t} \in A$ or $x_{t} q A$, then $x_{t} \in \vee q A$.

Definition 10. Let $A=<\mu_{A}, \nu_{A}>$ be intuitionistic fuzzy subset of $X$ and $t \in[0,1]$, then $t$-quasi-cut set, $t$-strong quasi cut set and $\in \vee q$ cut set. of fuzzy membership set $\mu_{A}$ are given by $\left(\mu_{A}\right)_{t}=\left\{x \mid x_{t} \in \mu_{A}\right\}=\left\{x \mid \mu_{A}(x) \geq t\right\}$

$\left(\mu_{A}\right)_{t}=\left\{x \mid x_{t} \in \mu_{A}\right\}=\left\{x \mid \mu_{A}(x)>t\right\}$,

$<\mu_{A}>_{t}=\left\{x \mid x_{t} q \mu_{A}\right\}=\left\{x \mid \mu_{A}(x)+t \geq 1\right\}$,

$<\mu_{A}>_{t}=\left\{x \mid x_{t} q \mu_{A}\right\}=\left\{x \mid \mu_{A}(x)+t>1\right\}$,

$\left[\mu_{A}\right]_{t}=\left\{x \mid x_{t} \in \vee q \mu_{A}\right\}=\left\{x \mid \mu_{A}(x) \geq t\right.$ or $\left.\mu_{A}(x)+t>1\right\}$, where $\left(\mu_{A}\right)_{t}$ is called $t$-cut set of $\mu_{A},\left(\mu_{A}\right)_{t}$ is called strong $t$-cut set of $\mu_{A},<\mu_{A}>_{t}$ is called $t$-quasi-cut set of $\mu_{A},<\mu_{A}>_{t}$ is called strong t-quasi-cut set of $\mu_{A}$ and $\left[\mu_{A}\right]_{t}$ is called $\in \vee q$-cut set of $\mu_{A}$, clearly $\left.\left[\mu_{A}\right]_{t}=<\mu_{A}\right\rangle_{t} \cup\left(\mu_{A}\right)_{t}$,

and $t$-quasi-cut set, $t$-strong quasi cut set and $\in \vee q$ cut set of fuzzy non membership set $\nu_{A}$ are given by

$\left(\nu_{A}\right)_{t}=\left\{x \mid x_{t} \in \nu_{A}\right\}=\left\{x \mid \nu_{A}(x) \leq t\right\}$,

$\left(\nu_{A}\right)_{t}=\left\{x \mid x_{t} \in \nu_{A}\right\}=\left\{x \mid \nu_{A}(x)<t\right\}$,

$<\nu_{A}>_{t}=\left\{x \mid x_{t} q \nu_{A}\right\}=\left\{x \mid \nu_{A}(x)+t \leq 1\right\}$,

$<\nu_{A}>_{t}=\left\{x \mid x_{t} q \nu_{A}\right\}=\left\{x \mid \nu_{A}(x)+t<1\right\}$,

$\left[\nu_{A}\right]_{t}=\left\{x \mid x_{t} \in \vee q \nu_{A}\right\}=\left\{x \mid \nu_{A}(x) \leq t\right.$ or $\left.\nu_{A}(x)+t<1\right\}$,

where $\left(\nu_{A}\right)_{t}$ is called $t$-cut set of $\nu_{A},\left(\nu_{A}\right)_{t}$ is called strong $t$-cut set of $\nu_{A},\left\langle\nu_{A}\right\rangle_{t}$ is called t-quasi-cut set of $\nu_{A},\left\langle\nu_{A}\right\rangle_{t}$ is called strong t-quasi-cut set of $\nu_{A}$ and $\left[\nu_{A}\right]_{t}$ is called $\in \vee q$ cut set of $\nu_{A}$, clearly $\left[\nu_{A}\right]_{t}=\left\langle\nu_{A}\right\rangle_{t} \cup\left(\nu_{A}\right)_{t}$.

Definition 11. Let $A=<\mu_{A}, \nu_{A}>$ be intuitionistic fuzzy subset of $X$ and $\alpha, \beta \in[0,1]$ then we define upper $(\alpha, \beta)$ cut set $A_{(\alpha, \beta)}$ and strong upper $(\alpha, \beta)$ cut set $A_{(\alpha, \beta)}$ of $A$ as

$$
\begin{aligned}
A_{(\alpha, \beta)} & =\left\{x \mid x \in X, \mu_{A}(x) \geq \alpha \quad \text { and } \quad \nu_{A}(x) \leq \beta\right\} \\
A_{(\alpha, \beta)} & =\left\{x \mid x \in X, \mu_{A}(x)>\alpha \quad \text { and } \quad \nu_{A}(x)<\beta\right\}
\end{aligned}
$$

Lower $(\alpha, \beta)$ cut set $A^{(\alpha, \beta)}$ and strong lower $(\alpha, \beta)$ cut set $A^{(\alpha, \beta)}$ as

$$
\begin{aligned}
A^{(\alpha, \beta)} & =\left\{x \mid x \in X, \mu_{A}(x) \leq \alpha \quad \& \quad \nu_{A}(x) \geq \beta\right\} \\
A^{(\alpha, \beta)} & =\left\{x \mid x \in X, \mu_{A}(x)<\alpha \quad \& \quad \nu_{A}(x)>\beta\right\}
\end{aligned}
$$

Definition 12. Let $A=<\mu_{A}, \nu_{A}>$ be intuitionistic fuzzy subset of $X$ and $\alpha, \beta \in[0,1]$ then we define upper $(\alpha, \beta)$-quasi-cut set $A_{<\alpha, \beta>}$, strong upper $(\alpha, \beta)$-quasi-cut set $A_{<\alpha, \beta>}$ of $A$ as

$$
\begin{aligned}
A_{<\alpha, \beta>} & =\left\{x \mid x \in X, \mu_{A}(x)+\alpha \geq 1 \quad \text { and } \quad \nu_{A}(x)+\beta \leq 1\right\} \\
A_{<\alpha, \beta>} & =\left\{x \mid x \in X, \mu_{A}(x)+\alpha>1 \quad \text { and } \quad \nu_{A}(x)+\beta<1\right\}
\end{aligned}
$$

Lower $(\alpha, \beta)$-quasi-cut set $A^{<\alpha, \beta>}$, strong lower $(\alpha, \beta)$-quasi-cut set $A<\alpha, \beta>$ as

$$
\begin{aligned}
& A^{<\alpha, \beta>}=\left\{x \mid x \in X, \mu_{A}(x)+\alpha \leq 1 \quad \& \quad \nu_{A}(x)+\beta \geq 1\right\} \\
& A^{<\alpha, \beta>}=\left\{x \mid x \in X, \mu_{A}(x)+\alpha<1 \quad \& \quad \nu_{A}(x)+\beta>1\right\}
\end{aligned}
$$

Definition 13. Let $A=<\mu_{A}, \nu_{A}>$ be intuitionistic fuzzy subset of $X$ and $t \in[0,1]$ then we define $(\in, \in \vee q)$-t-cut set of $A$ as

$$
\begin{aligned}
A_{t} & =\left\{x \mid x_{t} \in \vee q A\right\} \\
& =\left\{x \mid \mu_{A}(x) \geq t \text { and } \nu_{A}(x) \leq t \text { or } \mu_{A}(x)+t \geq 1 \text { and } \nu_{A}(x)+t \leq 1\right\}
\end{aligned}
$$




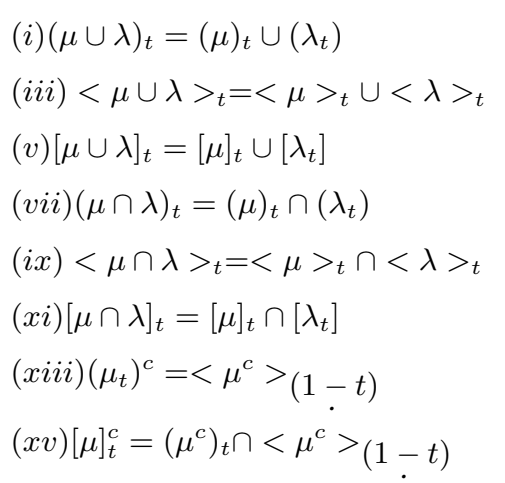

Proof. (i) We have $(\mu \cup \lambda)_{t}=\{x \in X \mid(\mu \cup \lambda)(x) \geq t\}$ Now $x \in(\mu \cup \lambda)_{t} \Leftrightarrow(\mu \cup \lambda)(x) \geq t$

$\Leftrightarrow \max \{\mu(x), \lambda(x)\} \geq t$

$\Leftrightarrow \mu(x) \geq t$ or $\lambda(x) \geq t$

$\Leftrightarrow x \in(\bar{\mu})_{t}$ or $x \in\left(\overline{\lambda_{t}}\right)$

Therefore $(\mu \cup \lambda)_{t}=(\mu)_{t} \cup\left(\lambda_{t}\right)$

(iv) we have $<\mu \cup \lambda>_{t}=\{x \in X \mid(\mu \cup \lambda)(x)+t>1\}$

Now $x \in<\mu \cup \lambda>_{t} \Leftrightarrow(\mu \cup \lambda)(x)+t>1$

$\Leftrightarrow \max \{\mu(x), \lambda(x) \dot{\}}+t>1$

$\Leftrightarrow \mu(x)+t>1$ or $\lambda(x)+t>1$

$\Leftrightarrow x \in<\mu>_{t}$ or $x \in<\lambda>_{t}$

Therefore $<\dot{\mu} \cup \lambda>_{t}=<\mu>_{t} \cup<\lambda_{t}>$

(ix) we have $<\mu \cap \lambda>_{t}=\{x \dot{\in} X \mid(\mu \dot{\cap} \lambda)(x)+t \geq 1\}$

Now $x \in<\mu \cap \lambda>_{t} \Leftrightarrow(\mu \cap \lambda)(x)+t \geq 1$

$\Leftrightarrow \min \{\mu(x), \lambda(x)\}+t \geq 1$

$\Leftrightarrow \mu(x)+t \geq 1$ and $\lambda(x)+t \geq 1$

$\Leftrightarrow x \in\langle\mu\rangle_{t}$ and $x \in<\lambda_{t}>$

Therefore $<\mu \cap \lambda>_{t}=\left\langle\mu>_{t} \cap<\lambda_{t}\right\rangle$

(xi)

we have $[\mu \cap \lambda]_{t}=\{x \in X \mid(\mu \cap \lambda)(x) \geq t$ or $(\mu \cap \lambda)(x)+t>1\}$

Now $x \in[\mu \cap \dot{\lambda}]_{t} \Leftrightarrow(\mu \cap \lambda)(x) \geq t$ or $\left.(\mu \cap \lambda)(x)+t>1\right\}$

$\Leftrightarrow \min \{\mu(x), \lambda(x)\} \geq t$ or $\min \{\mu(x), \lambda(x)\}+t>1$

$\Leftrightarrow \mu(x) \geq t, \lambda(x) \geq t$ or $\mu(x)+t>1, \lambda(x)+t>1$

$\Leftrightarrow \mu(x) \geq t$ or $\mu(x)+t>1$ and $\lambda(x) \geq t$ or $\lambda(x)+t>1$

$\Leftrightarrow x \in[\mu]_{t}$ and $x \in[\lambda]_{t}$

Therefore $[\mu \cap \lambda]_{t}=[\mu]_{t} \cap\left[\lambda_{t}\right]$

(xiii) we have

$$
\begin{aligned}
\left(\mu_{t}\right)^{c} & =\{x \mid \mu(x)+t \geq 1\}^{c} \\
& =\{x \mid \mu(x)+t<1\} \\
& =\{x \mid 1-\mu(x)>t\} \\
& =\{x \mid 1-\mu(x)+1-t>1\} \\
& =\left\{x \mid \mu^{c}(x)+1-t>1\right\} \\
& =<\mu^{c}>(1-t) \\
\Rightarrow\left(\mu_{t}\right)^{c} & =<\mu^{c}>(1-t)
\end{aligned}
$$

$$
\begin{aligned}
x \in[\mu]_{t}^{c} & \Leftrightarrow x \notin[\mu]_{t} \\
& \Leftrightarrow x_{t} \overline{\in \vee} \mu \\
& \Leftrightarrow x_{t} \bar{\epsilon} \mu \text { and } x_{t} \bar{q} \mu \\
& \Leftrightarrow \mu(x)<t \quad \text { and } \mu(x)+t \leq 1 \\
& \Leftrightarrow-\mu(x)>-t \quad \text { and }-\mu(x)-t \geq-1 \\
& \Leftrightarrow 1-\mu(x)>1-t \quad \text { and } \quad 1-\mu(x)-t \geq 0 \\
& \Leftrightarrow \mu^{c}(x)>1-t \quad \text { and } \mu^{c}(x)-t \geq 0 \\
& \Leftrightarrow \mu^{c}(x)>1-t \quad \text { and } \mu^{c}(x) \geq t \\
& \Leftrightarrow x \in\left(\mu^{c}\right)_{(1-t)} \quad \text { and } \quad x \in\left(\mu^{c}\right)_{t} \\
& \Leftrightarrow x \in\left(\mu^{c}\right)_{(1-t)} \cap\left(\mu^{c}\right)_{t}
\end{aligned}
$$

Hence $[\mu]_{t}^{c}=\left(\mu^{c}\right)_{t} \cap<\mu^{c}>(1-t)$

(xvi) Let

$$
\begin{aligned}
x \in[\mu]_{t} & \Leftrightarrow x \in[\mu]_{t} \\
& \Leftrightarrow x_{t} \in \vee q \mu \\
& \Leftrightarrow x_{t} \in \mu \quad \text { or } \quad x_{t} q \mu \\
& \Leftrightarrow \mu(x) \geq t \quad \text { or } \quad \mu(x)+t>1 \\
& \Leftrightarrow x \in(\mu)_{t} \text { or } \quad x \in<>_{t} \\
& \Leftrightarrow x \in(\mu)_{t} \cup \mu>_{t}
\end{aligned}
$$

Hence $[\mu]_{t}=<\mu>_{t} \cup(\mu)_{t}$

\section{THEOREM 15. If $A \subseteq B$ then}

$$
\begin{aligned}
\text { (i) } & A_{(\alpha, \beta)} \subseteq B_{(\alpha, \beta)} \\
\text { (ii) } & A_{(\alpha, \beta)} \subseteq B_{(\alpha, \beta)}
\end{aligned}
$$

Proof. If $A \subseteq B$ then

$$
\mu_{A}(x) \leq \mu_{B}(x) \operatorname{and} \nu_{A}(x) \geq \nu_{B}(x)
$$

(i) Let $x \in A_{(\alpha, \beta)}$

$$
\begin{array}{ll}
\Rightarrow \quad \mu_{A}(x) \geq \alpha \quad \text { and } \quad \nu_{A}(x) \leq \beta \\
\Rightarrow \quad \mu_{B}(x) \geq \alpha \quad \text { and } \quad \nu_{B}(x) \leq \beta \quad \text { by (3) } \\
\Rightarrow \quad x \in B_{(\alpha, \beta)}
\end{array}
$$

Therefore $x \in A_{(\alpha, \beta)} \Rightarrow x \in B_{(\alpha, \beta)}$

Hence $\quad A_{(\alpha, \beta)} \subseteq B_{(\alpha, \beta)}$

(ii) Same as (i) 
THEOREM 16. Let $A=<\mu_{A}, \nu_{A}>$ be intuitionistic fuzzy subset of $X$ and $\alpha, \beta \in[0,1]$ then

$$
\begin{aligned}
& (i)(A \cup B)_{(\alpha, \beta)} \supseteq A_{(\alpha, \beta)} \cup B_{(\alpha, \beta)} \\
& (i i)(A \cup B)_{(\alpha, \beta)} \supseteq A_{(\alpha, \beta)} \cup B_{(\alpha, \beta)} \\
& (i i i)(A \cup B)_{(\alpha, \beta)} \supseteq A_{(\alpha, \beta)} \cap B_{(\alpha, \beta)} \\
& (i v)(A \cup B)_{(\alpha, \beta)} \supseteq A_{(\alpha, \beta)} \cap B_{(\alpha, \beta)} \\
& (v)(A \cap B)_{(\alpha, \beta)}=A_{(\alpha, \beta)} \cap B_{(\alpha, \beta)} \\
& (v i)(A \cap B)_{(\alpha, \beta)}=A_{(\alpha, \beta)} \cap B_{(\alpha, \beta)} \\
& (v i i)\left(A^{c}\right)_{(\alpha, \beta)} \subseteq\left(A_{(\beta, \alpha)}\right)^{c} \\
& (v i i i)\left(A^{c}\right)_{(\alpha, \beta)} \subseteq\left(A_{(\beta, \alpha)}\right)^{c} \\
& (i x) \alpha \geq \gamma \text { and } \beta \leq \delta \Rightarrow A_{(\alpha, \beta)} \subseteq A_{(\gamma, \delta)} \\
& (x) \alpha \geq \gamma \text { and } \beta \leq \delta \Rightarrow A_{(\alpha, \beta)} \subseteq A_{(\gamma, \delta)}
\end{aligned}
$$

Proof. Since $A \subseteq A \cup B$ and $B \subseteq A \cup B$ Therefore by Theorem 15

(i) $A_{(\alpha, \beta)} \subseteq(A \cup B)_{(\alpha, \beta)}$ and $B_{(\alpha, \beta)} \subseteq(A \cup B)_{(\alpha, \beta)}$, and therefore

$(A \cup B)_{(\alpha, \beta)} \supseteq A_{(\alpha, \beta)} \cup B_{(\alpha, \beta)}$

(ii) $A_{(\alpha, \beta)} \subseteq(A \cup B)_{(\alpha, \beta)}$ and $B_{(\alpha, \beta)} \subseteq(A \cup B)_{(\alpha, \beta)}$, and therefore

$(A \cup B)_{(\alpha, \beta)} \supseteq A_{(\alpha, \beta)} \cup B_{(\alpha, \beta)}$

(vi) We have $(A \cap \dot{B})_{(\alpha, \beta)}=\left\{x \in X \mid\left(\mu_{A} \cap \mu_{B}\right)(x)>\right.$ $\left.\alpha,\left(\nu_{A} \cup \nu_{B}\right)(x)<\beta\right\}$

$$
\begin{aligned}
& x \in(A \cap B)(\alpha, \beta) \\
& \Leftrightarrow\left(\mu_{A} \cap \mu_{B}\right)(x)>\alpha \text { and }\left(\nu_{A} \cup\right. \\
& \Leftrightarrow \min \left\{\mu_{A}(x), \mu_{B}(x)\right\}>\alpha \text { and } \\
& \Leftrightarrow \mu_{A}(x)>\alpha, \mu_{B}(x)>\alpha \text { and } \nu_{A} \\
& \Leftrightarrow \mu_{A}(x)>\alpha, \nu_{A}(x)<\beta \text { and } \mu_{B} \\
& \Leftrightarrow x \in A_{(\alpha, \beta)} \text { and } x \in B_{(\alpha, \beta)} \\
& \Leftrightarrow x \in A_{(\alpha, \beta)} \cap B_{(\alpha, \beta)}
\end{aligned}
$$$$
\Leftrightarrow\left(\mu_{A} \cap \mu_{B}\right)(x)>\alpha \text { and }\left(\nu_{A} \cup \nu_{B}\right)(x)<\beta
$$$$
\Leftrightarrow \min \left\{\mu_{A}(x), \mu_{B}(x)\right\}>\alpha \text { and } \max \left\{\nu_{A}(x), \nu_{B}(x)\right\}<\beta
$$$$
\Leftrightarrow \mu_{A}(x)>\alpha, \mu_{B}(x)>\alpha \text { and } \nu_{A}(x)<\beta, \nu_{B}(x)<\beta
$$$$
\Leftrightarrow \mu_{A}(x)>\alpha, \nu_{A}(x)<\beta \text { and } \mu_{B}(x)>\alpha, \nu_{B}(x)<\beta
$$

Therefore $(A \cap B)_{(\alpha, \beta)}=A_{(\alpha, \beta)} \cap B_{(\alpha, \beta)}$ (viii)

$$
\begin{aligned}
& x \in\left(A^{c}\right)(\alpha, \beta) \\
& \Rightarrow \nu_{A}(x)>\alpha \text { and } \mu_{A}(x)<\beta \\
& \Rightarrow \mu_{A}(x)<\beta \text { and } \nu_{A}(x)>\alpha \\
& \Rightarrow \mu_{A}(x) \nsupseteq \beta \text { and } \nu_{A}(x) \not \leq \alpha \\
& \Rightarrow x \in\left(A_{(\beta, \alpha)}\right)^{c}
\end{aligned}
$$

Hence $\left(A^{c}\right)_{(\alpha, \beta)} \subseteq\left(A_{(\beta, \alpha)}\right)^{c}$

(ix) Here $\alpha \geq \dot{\gamma}$ and $\beta \leq \delta$

$$
\begin{aligned}
& x \in A_{(\alpha, \beta)} \\
& \Rightarrow \mu_{A}(x) \geq \alpha \text { and } \nu_{A}(x) \leq \beta \\
& \Rightarrow \mu_{A}(x) \geq \alpha \geq \gamma \text { and } \nu_{A}(x) \leq \beta \leq \delta \\
& \Rightarrow \mu_{A}(x) \geq \gamma \text { and } \nu_{A}(x) \leq \delta \\
& \Rightarrow x \in A_{(\gamma, \delta)}
\end{aligned}
$$

Hence $A_{(\alpha, \beta)} \subseteq A_{(\gamma, \delta)}$

\section{THEOREM 17. If $A \subseteq B$ then}

$$
\begin{aligned}
& \text { (i) } \quad A^{(\alpha, \beta)} \supseteq B^{(\alpha, \beta)} \\
& \text { (ii) } \quad A^{(\alpha, \beta)} \supseteq B^{(\alpha, \beta)}
\end{aligned}
$$

Proof. Same as above

THEOREM 18. Let $A=<\mu_{A}, \nu_{A}>$ be intuitionistic fuzzy subset of $X$ and $\alpha, \beta \in[0,1]$ then

$$
\begin{aligned}
& (i)(A \cup B)^{(\alpha, \beta)}=A^{(\alpha, \beta)} \cap B^{(\alpha, \beta)} \\
& (i i)(A \cup B)^{(\alpha, \beta)}=A^{(\alpha, \beta)} \cap B^{(\alpha, \beta)} \\
& (\text { iii })(A \cap B)^{(\alpha, \beta)} \supseteq A^{(\alpha, \beta)} \cup B^{(\alpha, \beta)} \\
& (i v)(A \cap B)^{(\alpha, \beta)} \supseteq A^{(\alpha, \beta)} \cup B^{(\alpha, \beta)} \\
& (v)\left(A^{c}\right)^{(\alpha, \beta)} \subseteq\left(A^{(\beta, \alpha)}\right)^{c} \\
& (v i)\left(A^{c}\right)^{(\alpha, \beta)} \subseteq\left(A^{(\beta, \alpha)}\right)^{c} \\
& (v i i) \alpha \geq \gamma \text { and } \beta \leq \delta \Rightarrow A^{(\alpha, \beta)} \supseteq A^{(\gamma, \delta)} \\
& (\text { viii }) \alpha \geq \gamma \text { and } \beta \leq \delta \Rightarrow A^{(\alpha, \beta)} \supseteq A^{(\gamma, \delta)}
\end{aligned}
$$

Proof. (i) Let

$$
\begin{aligned}
& x \in(A \cup B)^{(\alpha, \beta)} \\
& \Leftrightarrow\left(\mu_{A} \cup \mu_{B}\right)(x) \leq \alpha \text { and }\left(\nu_{A} \cap \nu_{B}\right)(x) \geq \beta \\
& \Leftrightarrow \max \left\{\mu_{A}(x), \mu_{B}(x)\right\} \leq \alpha \text { and } \min \left\{\nu_{A}(x), \nu_{B}(x)\right\} \geq \beta \\
& \Leftrightarrow \mu_{A}(x) \leq \alpha, \mu_{B}(x) \leq \alpha \text { and } \nu_{A}(x) \geq \beta, \nu_{B}(x) \geq \beta \\
& \Leftrightarrow \mu_{A}(x) \leq \alpha, \nu_{A}(x) \geq \beta \text { and } \mu_{B}(x) \leq \alpha, \nu_{B}(x) \geq \beta \\
& \Leftrightarrow x \in A^{(\alpha, \beta)} \text { and } x \in B^{(\alpha, \beta)} \\
& \Leftrightarrow x \in A^{(\alpha, \beta)} \cap B^{(\alpha, \beta)}
\end{aligned}
$$

Hence $(A \cup B)^{(\alpha, \beta)}=A^{(\alpha, \beta)} \cap B^{(\alpha, \beta)}$

(iii) Let

$$
\begin{aligned}
& x \in A^{(\alpha, \beta)} \cup B^{(\alpha, \beta)} \\
& \Rightarrow x \in A^{(\alpha, \beta)} \text { or } x \in B^{(\alpha, \beta)} \\
& \Rightarrow \mu_{A}(x) \leq \alpha \quad \text { and } \quad \nu_{A}(x) \geq \beta \quad \text { or } \quad \mu_{B}(x) \leq \alpha \quad \text { and } \quad \nu_{B}(x) \geq \\
& \Rightarrow \mu_{A}(x) \leq \alpha \quad \text { or } \quad \mu_{B}(x) \leq \alpha \quad \text { and } \quad \nu_{A}(x) \geq \beta \quad \text { or } \nu_{B}(x) \geq \beta \\
& \Rightarrow \min \left\{\mu_{A}(x), \mu_{B}(x)\right\} \leq \alpha \quad \text { and } \max \left\{\nu_{A}(x), \nu_{B}(x)\right\} \geq \beta \\
& \Rightarrow\left(\mu_{A} \cap \mu_{B}\right)(x) \leq \alpha \quad \text { and } \quad\left(\nu_{A} \cup \nu_{B}\right)(x) \geq \beta \\
& \Rightarrow x \in(A \cap B)^{(\alpha, \beta)}
\end{aligned}
$$

Hence $A^{(\alpha, \beta)} \cup B^{(\alpha, \beta)} \subseteq(A \cap B)^{(\alpha, \beta)}$

\section{THEOREM 19. If $A \subseteq B$ then}

$$
\begin{array}{ll}
\text { (i) } & A_{<\alpha, \beta>} \subseteq B_{<\alpha, \beta>} \\
\text { (ii) } & A_{<\alpha, \beta>} \subseteq B_{<\alpha, \beta>}
\end{array}
$$

Proof. Same as above 
THEOREM 20. Let $A=<\mu_{A}, \nu_{A}>$ be intuitionistic fuzzy subset of $X$ and $\alpha, \beta \in[0,1]$ then

$$
\begin{aligned}
& \text { (i) }(A \cup B)_{<\alpha, \beta>} \supseteq A_{<\alpha, \beta>} \cup B_{<\alpha, \beta>}
\end{aligned}
$$

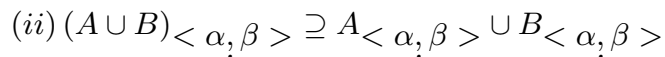

$$
\begin{aligned}
& \text { (iii) }(A \cap B)_{<\alpha, \beta>}=A_{<\alpha, \beta>} \cap B_{<\alpha, \beta>} \\
& \text { (iv) }(A \cap B)_{<\alpha, \beta>}=A_{<\alpha, \beta>} \cap B_{<\alpha, \beta>} \\
& (v)\left(A^{c}\right)_{<\alpha, \beta>} \subseteq\left(A_{<\beta, \alpha>}\right)^{c} \\
& (v i)\left(A^{c}\right)_{<\alpha, \beta>\subseteq\left(A_{<\beta, \alpha>}\right)^{c}} \\
& \text { (vii) } \alpha \geq \gamma \text { and } \beta \leq \delta \Rightarrow A_{<\alpha, \beta>} \supseteq A_{<\gamma, \delta>} \\
& \text { (viii) } \alpha \geq \gamma \text { and } \beta \leq \delta \Rightarrow A_{<\alpha, \beta>} \supseteq A_{<\gamma, \delta>}
\end{aligned}
$$

Proof. Since $A \subseteq A \cup B$ and $B \subseteq A \cup B$ Therefore by Theorem

(i) $A_{<\alpha, \beta>} \subseteq(A \cup B)_{<\alpha, \beta>}$ and $B_{<\alpha, \beta>} \subseteq(A \cup B)_{<\alpha, \beta>}$, and therefore

$(A \cup B)_{<\alpha, \beta>} \supseteq A_{<\alpha, \beta>} \cup B_{<\alpha, \beta>}$

(ii) $A_{<\alpha, \beta>} \subseteq(A \cup B)_{<\alpha, \beta>}$ and $B_{<\alpha, \beta>} \subseteq(A \cup$

$B)<\alpha, \beta>$, and therefore

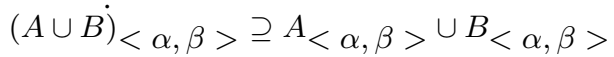

(iv) We have $(A \cap B)<\alpha, \dot{\beta}>=\left\{x \in \dot{X} \mid\left(\mu_{A} \cap \mu_{B}\right)(x)+\alpha>\right.$

$\left.1,\left(\nu_{A} \cup \nu_{B}\right)(x)+\beta<1\right\}$

$$
\begin{aligned}
& x \in(A \cap B)_{<\alpha, \beta>} \\
& \Leftrightarrow\left(\mu_{A} \cap \mu_{B}\right)(x)+\alpha>1 \text { and }\left(\nu_{A} \cup \nu_{B}\right)(x)+\beta<1 \\
& \Leftrightarrow \min \left\{\mu_{A}(x), \mu_{B}(x)\right\}+\alpha>1 \text { and } \max \left\{\nu_{A}(x), \nu_{B}(x)\right\}+\beta<1 \\
& \Leftrightarrow \mu_{A}(x)+\alpha>1, \mu_{B}(x)+\alpha>1 \text { and } \nu_{A}(x)+\beta<1, \nu_{B}(x)+\beta<1 \\
& \Leftrightarrow \mu_{A}(x)+\alpha>1, \nu_{A}(x)+\beta<1 \text { and } \mu_{B}(x)+\alpha>1, \nu_{B}(x)+\beta<1 \\
& \Leftrightarrow x \in A_{<\alpha, \beta>\text { and } x \in B_{<\alpha, \beta>}} \\
& \Leftrightarrow x \in A_{<\alpha, \beta>} \cap B_{<\alpha, \beta>}
\end{aligned}
$$

Therefore $(A \cap B)_{<\alpha, \beta>}=A_{<\alpha, \beta>} \cap B_{<\alpha, \beta>}$

(vi)

$$
\begin{aligned}
& x \in\left(A^{c}\right)<\alpha, \beta> \\
& \Leftrightarrow \nu_{A}(x)+\alpha>1 \text { and } \mu_{A}(x)+\beta<1 \\
& \Leftrightarrow \mu_{A}(x)+\beta<1 \text { and } \nu_{A}(x)+\alpha>1 \\
& \Leftrightarrow \mu_{A}(x)+\beta \nsupseteq 1 \text { and } \nu_{A}(x)+\alpha \not \leq 1 \\
& \Leftrightarrow x \in\left(A_{<\beta, \alpha>}\right)^{c}
\end{aligned}
$$

Hence $\left(A^{c}\right)<\alpha, \beta>\subseteq\left(A_{<\beta, \alpha>}\right)^{c}$

(vii) Here $\alpha \geq \gamma$ and $\beta \leq \delta$

$$
\begin{aligned}
& \text { Let } \quad x \in A_{<\gamma, \delta>} \\
& \Rightarrow \mu_{A}(x)+\gamma \geq 1 \text { and } \nu_{A}(x)+\delta \leq 1 \\
& \Rightarrow \mu_{A}(x)+\alpha \geq 1 \text { and } \nu_{A}(x)+\beta \leq 1 \\
& \Rightarrow x \in A_{<\alpha, \beta>}
\end{aligned}
$$

Hence $A_{<\gamma, \delta>} \subseteq A_{<\alpha, \beta>}$
Theorem 21. If $A \subseteq B$ then

$$
\begin{aligned}
& \text { (i) } \quad A^{<\alpha, \beta>} \supseteq B^{<\alpha, \beta>} \\
& \text { (ii) } \quad A^{<\alpha, \beta>} \supseteq{ }^{<\alpha, \beta>}
\end{aligned}
$$

Proof. Same as above

THEOREM 22. Let $A=<\mu_{A}, \nu_{A}>$ be intuitionistic fuzzy subset of $X$ and $\alpha, \beta \in[0,1]$ then

$$
\begin{aligned}
& \text { (i) }(A \cup B)^{<\alpha, \beta>} \subseteq A^{<\alpha, \beta>} \cap B^{<\alpha, \beta>} \\
& \text { (ii) }(A \cup B)^{<\alpha, \beta>} \subseteq A^{<\alpha, \beta>} \cap B^{<\alpha, \beta>} \\
& \text { (iii) }(A \cap B)^{<\alpha, \beta>}=A^{<\alpha, \beta>} \cup B^{<\alpha, \beta>} \\
& \text { (iv) }(A \cap B)^{<\alpha, \beta>}=A^{<\alpha, \beta>} \cup B<\alpha, \beta> \\
& \text { (v) }\left(A^{c}\right)^{<\alpha, \beta>} \subseteq\left(A^{<\alpha, \beta>}\right)^{c} \\
& \text { (vi) }\left(A^{c}\right)^{<\alpha, \beta>} \subseteq\left(A^{<\beta, \alpha>}\right)^{c} \\
& \text { (vii) } \alpha \geq \gamma \text { and } \beta \leq \delta \Rightarrow A^{<\alpha, \beta>} \subseteq A^{<\gamma, \delta>} \\
& \text { (viii) } \alpha \geq \gamma \text { and } \beta \leq \delta \Rightarrow A^{<\alpha, \beta>} \subseteq A^{<\gamma, \delta>}
\end{aligned}
$$

Proof. Similar to Theorem 20

Theorem 23. If $A \subseteq B$ then $A_{t} \subseteq B_{t}$

PROOF. Straightforward.

THEOREM 24. Let $A=<\mu_{A}, \nu_{A}>$ be intuitionistic fuzzy subset of $X$ and $t \in[0,1]$, then $(A \cup B)_{t}=A_{t} \cup B_{t}$

PROOF. Straightforward.

THEOREM 25. Let $f: X \longrightarrow Y$ be a mapping, then

(i)f $f\left(\mu_{t}\right) \subseteq(f(\mu))_{t}$

(ii) $f\left(\mu_{t}\right) \subseteq(f(\mu))_{t}$

(iii) $f\left(<\mu>_{t}\right) \subseteq<f(\mu)>_{t}$

(iv) $f\left(<\mu>_{t}\right) \subseteq<f(\mu)>_{t}$

$\left.(v) f[\mu]_{t}\right) \subseteq[f(\mu)]_{t}$

$\left.(v i) f[\mu]_{t}\right) \subseteq[f(\mu)]_{t}$

$(v i i) f^{-1}\left(\mu_{t}\right)=\left(f^{-1}(\mu)\right)_{t}$

(viii) $f^{-1}\left(\mu_{t}\right)=\left(f^{-1}(\mu)\right)_{t}$

$(i x) f^{-1}\left(<\mu>_{t}\right)=<f^{-1}(\mu)>_{t}$

$(x) f^{-1}\left(<\mu>_{t}\right)=<f^{-1}(\mu)>_{t}$

$\left.(x i) f^{-1}[\mu]_{t}\right)=\left[f^{-1}(\mu)\right]_{t}$

$($ xii $\left.) f^{-1}[\mu]_{t}\right)=\left[f^{-1}(\mu)\right]_{t}$

Proof. (i) Let $y \in f\left(\mu_{t}\right)$ be any element, then there exists $x \in \mu_{t}$ such that $f(x)=y$ and $\mu(x) \geq t$

$\Rightarrow \vee\left\{\mu(x): x \in f^{-1}(y)\right\} \geq t$

$\Rightarrow f(\mu)(f(x)) \geq t$

$\Rightarrow f(\mu)(y) \geq t$

$\Rightarrow y \in(f(\mu))_{t}$

Hence $f\left(\mu_{t}\right) \subseteq(f(\mu))_{t}$

(iv) Let $y \in \bar{f}\left\langle\mu_{t}\right\rangle$ be any element, then there exists $x \in \mu_{t}$ such that $f(x)=y$ and $\mu(x)+t>1$ 
$\Rightarrow \vee\left\{\mu(x): x \in f^{-1}(y)\right\}+t>1$

$\Rightarrow f(\mu)(f(x))+t>1$

$\Rightarrow f(\mu)(y)+t>1$

$\Rightarrow y \in<f(\mu)>_{t}$

Hence $f\left(<\mu>_{t}\right) \subseteq<f(\mu)>_{t}$

(vii) we have

$$
\begin{aligned}
\left(f^{-1}(\mu)\right)_{t} & =\left\{x \in X \mid f^{-1}(\mu)(x) \geq t\right\} \\
& =\{x \in X \mid \mu f(x) \geq t\} \\
& =\left\{x \in X \mid f(x) \in \mu_{t}\right\} \\
& =\left\{x \in X \mid x \in f^{-1}\left(\mu_{t}\right)\right\} \\
& =f^{-1}\left(\mu_{t}\right)
\end{aligned}
$$

Hence $f^{-1}\left(\mu_{t}\right)=\left(f^{-1}(\mu)\right)_{t}$

(ix) we have

$$
\begin{aligned}
<f^{-1}(\mu)>_{t} & =\left\{x \in X \mid f^{-1}(\mu)(x)+t>1\right\} \\
& =\{x \in X \mid \mu f(x)+t>1\} \\
& =\left\{x \in X \mid f(x) \in<\mu_{t}>\right\} \\
& =\left\{x \in X \mid x \in f^{-1}<\mu_{t}>\right\} \\
& =f^{-1}\left(<\mu_{t}>\right)
\end{aligned}
$$

Hence $f^{-1}\left(<\mu_{t}>\right)=<f^{-1}(\mu)>_{t}$

THEOREM 26. Let $f: X \longrightarrow Y$ be a mapping, then

$$
\begin{aligned}
& \text { (i) } f\left(A_{(\alpha, \beta)}\right) \subseteq(f(A))_{(\alpha, \beta)} \\
& (x) f^{-1}\left(B_{(\alpha, \beta)}\right)=\left(f^{-1}(B)\right)_{(\alpha, \beta)} \\
& \text { (ii)f }\left(_{(\alpha, \beta)}\right) \subseteq(f(A))_{(\alpha, \beta)} \\
& (x i) f^{-1}\left(B_{(\alpha, \beta)}\right)=\left(f^{-1}(B)\right)_{(\alpha, \beta)} \\
& \text { (iii) } f\left(A^{(\alpha, \beta)}\right) \subseteq(f(A))^{(\alpha, \beta)} \\
& (x i i) f^{-1}\left(B^{(\alpha, \beta)}\right)=\left(f^{-1}(B)\right)^{(\alpha, \beta)} \\
& \text { (iv)f(A } \left.A^{(\alpha, \beta)}\right) \subseteq(f(A))^{(\alpha, \beta)} \\
& \left(\text { xiii) } f^{-1}\left(B^{(\alpha, \beta)}\right)=\left(f^{-1}(B)\right)^{(\alpha, \beta)}\right. \\
& (v) f\left(A_{<\alpha, \beta>}\right) \subseteq(f(A))_{<\alpha, \beta>} \\
& (\text { xiv }) f^{-1}\left(B_{<\alpha, \beta>}\right)=\left(f^{-1}(B)\right)_{<\alpha, \beta>} \\
& \text { (vi) } f\left(A_{<\alpha, \beta>}\right) \subseteq(f(A))_{<\alpha, \beta>} \\
& (x v) f^{-1}\left(B_{<\alpha, \beta>}\right)=\left(f^{-1}(B)\right)_{<\alpha, \beta>} \\
& \text { (vii) } f\left(A^{<\alpha, \beta>}\right) \subseteq(f(A))^{<\alpha, \beta>} \\
& (x v i) f^{-1}\left(B^{<\alpha, \beta>}\right)=\left(f^{-1}(B)\right)^{<\alpha, \beta>} \\
& \text { (viii) } f\left(A^{<\alpha, \beta>}\right) \subseteq(f(A))^{<\alpha, \beta>} \\
& \text { (xvii) } f^{-1}\left(B^{<\alpha, \beta>}\right)=\left(f^{-1}(B)\right)<\alpha, \beta> \\
& \text { (ix) } f\left(A_{t}\right) \subseteq(f(A))_{t}, \quad \forall A \in I F S(X) \\
& \text { (xviii) } f^{-1}\left(B_{t}\right)=\left(f^{-1}(B)\right)_{t}, \quad \forall B \in I F S(X)
\end{aligned}
$$

Proof. (v) Let $y \in f\left(A_{<\alpha, \beta>}\right)$ be any element, then there exists $x \in A_{<\alpha, \beta>}$ such that $f(x)=y$ and $\mu_{A}(x)+\alpha \geq 1$ and $\nu_{A}(x)+\beta \leq 1$

$\Rightarrow \vee\left\{\mu_{A}(x): x \in f^{-1}(y)\right\}+\alpha \geq 1$ and $\wedge\left\{\nu_{A}(x): x \in\right.$ $\left.f^{-1}(y)\right\}+\beta \leq 1$

$\Rightarrow \mu_{f(A)}(f(x))+\alpha \geq 1$ and $\nu_{f(A)}(f(x))+\beta \leq 1$

$\Rightarrow f(\mu)(y)+\alpha \geq 1$ and $f(\nu)(y)+\beta \leq 1$
$\Rightarrow y \in(f(A))_{<\alpha, \beta>}$

Hence $f\left(A_{<\alpha, \beta>}\right) \subseteq(f(A))_{<\alpha, \beta>}$

(xiv) we have

$$
\begin{aligned}
\left(f^{-1}(B)\right)_{<\alpha, \beta>} & =\left\{x \in X \mid \mu_{f^{-1}(B)}(x)+\alpha \geq 1, \nu_{f^{-1}(B)}(x)+\beta \leq 1\right\} \\
& =\left\{x \in X \mid \mu_{B} f(x)+\alpha \geq 1, \nu_{B} f(x)+\beta \leq 1\right\} \\
& =\left\{x \in X \mid f(x) \in B_{<\alpha, \beta>}\right\} \\
& =\left\{x \in X \mid x \in f^{-1}\left(B_{<\alpha, \beta>}\right)\right\} \\
& =f^{-1}\left(B_{<\alpha, \beta>}\right)
\end{aligned}
$$

Hence $f^{-1}\left(B_{<\alpha, \beta>}\right)=\left(f^{-1}(B)\right)_{<\alpha, \beta>}$

\section{CUT SET OF CARTESIAN PRODUCT OF FUZZY SETS AND INTUITIONISTIC FUZZY SETS}

DEFINITION 27. Let $\mu, \nu$ be two two fuzzy subsets of $X$ and $Y$ respectively then their cartesian product of $\mu$ and $\nu$ is denoted by $\mu \times \nu$ and is defined as $\mu \times \nu=\{(x, y),(\mu \times \nu)(x, y) \mid x \in X, y \in$ $Y\}$, where $(\mu \times \nu)(x, y)=\min \{\mu(x), \nu(y)\}$

THEOREM 28. Let $\mu, \nu$ be two two fuzzy subsets of $X$ and $Y$ respectively, then
(i) $(\mu \times \nu)_{t}=(\mu)_{t} \times(\nu)_{t}$
(ii) $(\mu \times \nu)_{t}=(\mu)_{t} \times(\nu)_{t}$
(iii) $<\mu \times \nu>_{t}=\left\langle\mu>_{t} \times<\nu>_{t}\right.$
(iv) $<\mu \times \nu>_{t}=<\mu>_{t} \times<\nu>_{t}$
(v) $[\mu \times \nu]_{t}=[\mu]_{t} \times[\nu]_{t}$
(vi) $[\mu \times \nu]_{t}=[\mu]_{\underline{t}} \times[\nu]_{\underline{t}}$

Proof. (i) Let $(x, y) \in(\mu \times \nu)_{t}$ be any element

$$
\begin{aligned}
& \Leftrightarrow \quad(\mu \times \nu)(x, y) \geq t \\
& \Leftrightarrow \quad \min \{\mu(x), \nu(y)\} \geq t \\
& \Leftrightarrow \quad \mu(x) \geq t, \nu(y) \geq t \\
& \Leftrightarrow \quad x \in(\mu)_{t}, y \in(\nu)_{t} \\
& \Leftrightarrow \quad(x, y) \in(\mu)_{t} \times(\nu)_{t}
\end{aligned}
$$

Hence $(\mu \times \nu)_{t}=(\mu)_{t} \times(\nu)_{t}$

(iii) Let $(x, y) \in\langle\mu \times \nu\rangle_{t}$ be any element

$$
\begin{aligned}
& \Leftrightarrow \quad(\mu \times \nu)(x, y)+t \geq 1 \\
& \Leftrightarrow \quad \min \{\mu(x), \nu(y)\}+t \geq 1 \\
& \Leftrightarrow \quad \mu(x)+t \geq 1, \nu(y)+t \geq 1 \\
& \Leftrightarrow \quad x \in<\mu>_{t}, y \in<\nu>_{t} \\
& \Leftrightarrow \quad(x, y) \in<\mu>_{t} \times<>_{t}
\end{aligned}
$$

Hence $\left.<\mu \times \nu\rangle_{t}=\langle\mu\rangle_{t} \times<\nu\right\rangle_{t}$

DEFINITION 29. In [10] there are six ways catesian product of two IFs are defined, here we use only two ways viz. $\times_{4}$ and $\times_{5}$. Let $A=\left(\mu_{A}, \nu_{A}\right)$ and $B=\left(\mu_{B}, \nu_{B}\right)$ be any two IFSs of $X$ and $Y$ respectively. Then their cartesian product $A \times_{4} B$ is defined by $\left(A \times_{4} B\right)(x, y)=$ $\left\{<(x, y), \mu_{\left(A \times{ }_{4} B\right)}(x, y), \nu_{\left(A \times{ }_{4} B\right)} \quad>: \quad x, y \in X\right\}$ where $\mu_{\left(A \times_{4} B\right)}(x, y)=\min \left\{\mu_{A}(x), \mu_{B}(y)\right\}, \nu_{\left(A \times_{4} B\right)}(x, y)=$ $\max \left\{\nu_{A}(x), \nu_{B}(y)\right\}$ and their cartesian product $A \quad \times_{5} \quad B \quad$ is defined by $\left(\begin{array}{llll}A & \times_{5} & B\end{array}\right)(x, y)=\{<$ $\left.\left.(x, y), \mu_{(A \times 5 B)}(x, y), \nu_{(A \times 5} B\right) \quad>: \quad x, y \in X\right\}$ where 

$\begin{aligned} & \mu_{\left(A \times_{5} B\right)}(x, y)= \\ & \min \left\{\nu_{A}(x), \nu_{B}(y)\right\}\end{aligned} \quad \max \left\{\mu_{A}(x), \mu_{B}(y)\right\}, \nu_{\left(A \times_{5} B\right)}(x, y)=$

THEOREM 30. Let $A=\left(\mu_{A}, \nu_{A}\right)$ and $B=\left(\mu_{B}, \nu_{B}\right)$ be any two IFSs of of $X$ and $Y$ respectively, then

$$
\begin{aligned}
& \text { (i) }\left(A \times_{4} B\right)_{(\alpha, \beta)}=A_{(\alpha, \beta)} \times_{4} B_{(\alpha, \beta)} \\
& \text { (ii) }\left(A \times_{4} B\right)_{(\alpha, \beta)}=A_{(\alpha, \beta)} \times{ }_{4} B_{(\alpha, \beta)} \\
& \text { (iii) }\left(A \times{ }_{4} B\right)^{(\alpha, \beta)} \subseteq A^{(\alpha, \beta)} \times_{4} B^{(\alpha, \beta)} \\
& \text { (iv) }\left(A \times_{4} B\right)^{(\alpha, \beta)} \subseteq A^{(\alpha, \beta)} \times_{4} B^{(\alpha, \beta)} \\
& \text { (v) }\left(A \times_{4} B\right)_{<\alpha, \beta>}=A_{<\alpha, \beta>} \times{ }_{4} B_{<\alpha, \beta>} \\
& \text { (vi) }\left(A \times{ }_{4} B\right)_{<\alpha, \beta>}=A_{<\alpha, \beta>} \times{ }_{4} B_{<\alpha, \beta>} \\
& \text { (vii) }\left(A \times{ }_{4} B\right)^{<\alpha, \beta>} \subseteq A^{<\alpha, \beta>} \times{ }_{4} B^{<\alpha, \beta>} \\
& \text { (viii) }\left(A \times_{4} B\right)^{<\alpha, \beta>} \subseteq A^{<\alpha, \beta>} \times_{{ }_{4} B}<\alpha, \beta> \\
& (i x)\left(A \times_{5} B\right)_{(\alpha, \beta)} \subseteq A_{(\alpha, \beta)} \times_{5} B_{(\alpha, \beta)} \\
& (x)\left(A \times_{5} B\right)_{(\alpha, \beta)} \subseteq A_{(\alpha, \beta)} \times_{5} B_{(\alpha, \beta)} \\
& (x i)\left(A \times_{5} B\right)^{(\alpha, \beta)}=A^{(\alpha, \beta)} \times_{5} B^{(\alpha, \beta)} \\
& (x i i)\left(A \times_{5} B\right)^{(\alpha, \beta)}=A^{(\alpha, \beta)} \times_{5} B^{(\alpha, \beta)} \\
& \text { (xiii) }\left(A \times_{5} B\right)_{<\alpha, \beta>} \subseteq A_{<\alpha, \beta>} \times_{5} B_{<\alpha, \beta>}
\end{aligned}
$$

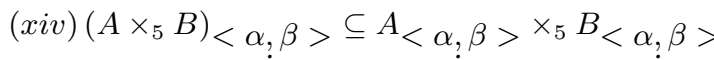

$$
\begin{aligned}
& (x v)\left(A \times_{5} B\right)^{<\alpha, \beta>}=A^{<\alpha, \beta>} \times_{5} B^{<\alpha, \beta>} \\
& (\text { xvi })\left(A \times_{5} B\right)^{<\alpha, \beta>}=A^{<\alpha, \beta>} \times_{{ }_{5} B}<\alpha, \beta> \\
& \text { (xvii) }\left(A \times_{4} B\right)_{t}=A_{t} \times_{4} B_{t} \\
& \text { (xviii) }\left(A \times_{5} B\right)_{t} \subseteq A_{t} \times_{5} B_{t}
\end{aligned}
$$

$\forall \alpha, \beta \in[0,1]$, with $0 \leq \alpha+\beta \leq 1$

Proof. (i)Let $(x, y) \in\left(A \times_{4} B\right)_{(\alpha, \beta)}$ be any element

$$
\begin{array}{ll}
\Leftrightarrow & \mu_{\left(A \times{ }_{4} B\right)}(x, y) \geq \alpha \quad \text { and } \quad \nu_{\left(A \times{ }_{4} B\right)}(x, y) \leq \beta \\
\Leftrightarrow & \min \left\{\mu_{A}(x), \mu_{B}(y)\right\} \geq \alpha \quad \text { and } \max \left\{\nu_{A}(x), \nu_{B}(y)\right\} \leq \beta \\
\Leftrightarrow & \mu_{A}(x) \geq \alpha, \mu_{B}(y) \geq \alpha \quad \text { and } \quad \nu_{A}(x) \leq \beta, \nu_{B}(y) \leq \beta \\
\Leftrightarrow & \mu_{A}(x) \geq \alpha, \nu_{A}(x) \leq \beta \quad \text { and } \quad \mu_{B}(y) \geq \alpha, \nu_{B}(y) \leq \beta \\
\Leftrightarrow & x \in A_{(\alpha, \beta)} \text { and } y \in B_{(\alpha, \beta)} \\
\Leftrightarrow & (x, y) \in A_{(\alpha, \beta)} \times_{4} B_{(\alpha, \beta)}
\end{array}
$$

Hence $\left(A \times_{4} B\right)_{(\alpha, \beta)}=A_{(\alpha, \beta)} \times_{4} B_{(\alpha, \beta)}$

(iv) Let $(x, y) \in\left(A \times{ }_{4} B\right)^{(\alpha, \beta)}$ be any element

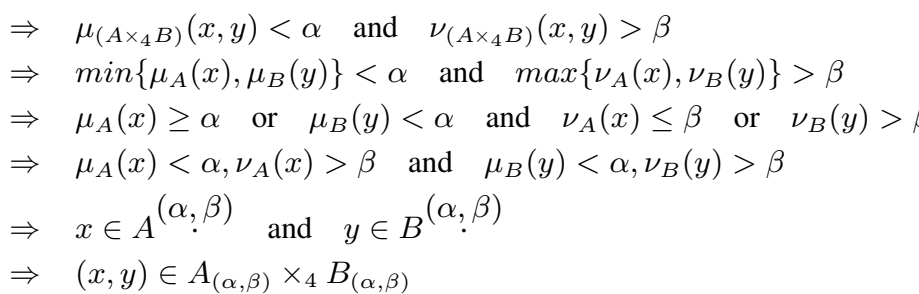

Hence $\left(A \times_{4} B\right)^{(\alpha, \beta)} \subseteq A^{(\alpha, \beta)} \times_{4} B^{(\alpha, \beta)}$

(ix)Let $(x, y) \in\left(A \times_{5} \bar{B}\right)_{(\alpha, \beta)}$ be any element

$$
\begin{aligned}
& \Rightarrow \mu_{(A \times 5)}(x, y) \geq \alpha \text { and } \nu_{\left(A \times{ }_{5} B\right)}(x, y) \leq \beta \\
& \Rightarrow \max \left\{\mu_{A}(x), \mu_{B}(y)\right\} \geq \alpha \text { and } \min \left\{\nu_{A}(x), \nu_{B}(y)\right\} \leq \beta \\
& \Rightarrow \mu_{A}(x) \geq \alpha \text { or } \mu_{B}(y) \geq \alpha \text { and } \nu_{A}(x) \leq \beta \text { or } \nu_{B}(y) \leq \beta \\
& \Rightarrow \quad \mu_{A}(x) \geq \alpha, \nu_{A}(x) \leq \beta \text { and } \mu_{B}(y) \geq \alpha, \nu_{B}(y) \leq \beta \\
& \Rightarrow \quad x \in A_{(\alpha, \beta)} \text { and } y \in B_{(\alpha, \beta)} \\
& \Rightarrow(x, y) \in A_{(\alpha, \beta)} \times{ }_{5} B_{(\alpha, \beta)}
\end{aligned}
$$

Hence $\left(A \times_{4} B\right)_{(\alpha, \beta)} \subseteq A_{(\alpha, \beta)} \times{ }_{4} B_{(\alpha, \beta)}$

(xii) Let $(x, y) \in\left(A \times_{5} B\right)^{(\alpha, \beta)}$ be any element

$$
\begin{aligned}
& \Leftrightarrow \quad \mu_{\left(A \times{ }_{5} B\right)}(x, y)<\alpha \quad \text { and } \nu_{\left(A \times{ }_{5} B\right)}(x, y)>\beta \\
& \Leftrightarrow \quad \max \left\{\mu_{A}(x), \mu_{B}(y)\right\}<\alpha \quad \text { and } \min \left\{\nu_{A}(x), \nu_{B}(y)\right\}>\beta \\
& \Leftrightarrow \quad \mu_{A}(x) \geq \alpha, \mu_{B}(y)<\alpha \quad \text { and } \nu_{A}(x) \leq \beta, \nu_{B}(y)>\beta \\
& \Leftrightarrow \quad \mu_{A}(x)<\alpha, \nu_{A}(x)>\beta \quad \text { and } \mu_{B}(y)<\alpha, \nu_{B}(y)>\beta \\
& \Leftrightarrow \quad x \in A^{(\alpha, \beta) \quad \text { and } y \in B}(\alpha, \beta) \\
& \Leftrightarrow \quad(x, y) \in A_{(\alpha, \beta)} \times_{5} B_{(\alpha, \beta)}
\end{aligned}
$$

Hence $\left(A \times_{5} B\right)^{(\alpha, \beta)}=A^{(\alpha, \beta)} \times_{5} B^{(\alpha, \beta)}$

\section{CONCLUSION}

In this paper, we have discussed detail theory of cut sets in fuzzy sets and in intuitionistic fuzzy sets. It is observed that the papers [4, 6] 3] and [7] are purely based on cut sets. Now our expectation is that this work will built foundations for further study of the theory of cut sets in both fuzzy sets and intuitionistic fuzzy sets. Also, in our opinion, the definition of various types of cut sets can be extended to cut sets of interval-valued fuzzy sets and cubic fuzzy sets.

\section{REFERENCES}

[1] L. A. Zadeh, Fuzzy sets, Information and Control 8 (1965), 338-353.

[2] K. T. Atanassov, Intuitionistic Fuzzy Sets, VII ITKR's Session, Sofia,(Deposed in Central Sci. - Techn. Library of Bulg. Acad. of Sci., 1697/84) (June 1983)(in Bulg.)

[3] P. S. Das, Fuzzy groups and level subgroups, J. Math. Anal. Appl. 85(1981) 264-269.

[4] D. K. Basnet, ( $\alpha, \beta)$-Cut of Intuitionistic Fuzzy Ideals, International Journal of Algebra, 4(27) (2010), 1329-1334.

[5] S. K. Bhakat, P. Das, $(\in, \in \vee q)$-fuzzy subgroup, Fuzzy Sets and Systems 80 (1996) 359-368.

[6] S. K. Bhakat, P. Das, $(\in, \in \vee q)$-level subset, Fuzzy sets and systems, 103(3), (1999), 529-533.

[7] P. K. Sharma, $(\alpha, \beta)$-Cut of Intuitionistic Fuzzy Groups, International Mathematical Forum, 6(53) (2011), 2605-2614.

[8] L. C. Zhong, Introduction to fuzzy (I), (II),Beijing Normal University Press (1989),(1993).

[9] K. T. Atanassov, Intuitionistic Fuzzy Sets , Fuzzy sets and Systems.vol.1(1986), 87-96

[10] K. T. Atanassov, On Intuitionistic Fuzzy Sets Theory, Published by Springer-Verlag Berlin Heidelberg, 2012. 
[11] P. P. Ming, L. Y. Ming, Fuzzy topology I: neighbourhood structure of a fuzzy point and Moore-Smith convergence,, J. Math. Anal. Appl. 76(1980) 571-599.

[12] V. Murali, Fuzzy points of equivalent fuzzy subsets, inform Sci, 158 (2004) 277-288. 